\title{
On Possibility of Geometrical Interpretation of Time
}

\author{
Oleg A. Olkhov \\ Laboratory of Theoretical Physics, Institute of Chemical Physics of Russian Academy of Sciences, Moscow, Russia \\ Email: oleg.olkhov@rambler.ru
}

How to cite this paper: Olkhov, O.A. (2017) On Possibility of Geometrical Interpretation of Time. Journal of High Energy Physics, Gravitation and Cosmology, 3, 173-177.

https://doi.org/10.4236/jhepgc.2017.32018

Received: December 5, 2016

Accepted: March 3, 2017

Published: March 6, 2017

Copyright $\odot 2017$ by author and Scientific Research Publishing Inc. This work is licensed under the Creative Commons Attribution International License (CC BY 4.0).

http://creativecommons.org/licenses/by/4.0/ (c) (i) Open Access

\begin{abstract}
The low-dimensional model of the space-time is considered where time is a real coordinate with dimensionality of length. The inertia law appears within this model as a consequence of the geometrical structure of the space.
\end{abstract}

\section{Keywords}

Special Relativity, Space of Events, Time and Geometry

\section{Introduction}

The time is considered in Mechanics as one of the four coordinates $\left(t, x_{1}, x_{2}, x_{3}\right)$ in the "space of events," where $t$ is the "moment of time" when the event takes place and $x_{1}, x_{2}, x_{3}$ are coordinates of the "place of event" [1] [2]. Hard sticks are standards for measuring of coordinates of "places of events" and periodical movements (clocks) are standards for measuring of "moments of time". Definition of standards means that the space of events can be investigated by physical methods, and it was established that three coordinates $x_{1}, x_{2}, x_{3}$ describe threedimensional Euclidean space. This space looks, intuitively, as understandable one because its standards have dimensionality of length. On the other hand, time does not look understandable on the basis of notions from everyday life. Here we show in the framework of the low-dimensional model that time can be also considered as coordinate with dimensionality of length in some special manydimensional space. It means that notions "space" and "time" can be reduced to the one notion-coordinates of some space with definite geometry.

The formal geometrical interpretation of time is known where time is considered as an imaginary fourth coordinate with the dimensionality of length in the four dimensional pseudo Euclidean space (Minkowski space) [1]. But representation of time by means of imaginary numbers does not give the opportunity to understand reasons of deep difference between notions "space" and "time". In- 
stead of Minkowski space, the geometrical model of the space of events is considered where all coordinates are real numbers. The space in this model is a topological product of the usual Euclidean space and some another space.

This work is a continuation of author's investigations of possibility to explain physical phenomena by geometrical properties of the space-time [3] [4] [5] [6]. In these investigations the idea was used about increasing dimensionality of our Euclidean space from three to four-the idea firstly used by Kaluza and then by Bergmann and Einstein in their attempts to geometrize electromagnetic field [7] [8]. The idea about additional dimensionalities is the main idea of this work, and this idea is based on the hypothesis that the space of events is a special case of the topological product of the usual Euclidean space and some space with the topology of closed manifold.

\section{Low-Dimensional Model of the Space-Time}

One of the simplest examples of topological products of spaces is shown at Figure 1. This is a surface of the usual cylinder that can be considered as topological product of one dimensional Euclidean space and one dimensional circle.

Topological products of spaces are a particular case of fiber spaces, and the theory of fiber spaces are now in the course of development [2]. The object of this presentation is to show principal possibility of geometrical interpretation of time, and for this purpose we suggest here the simplest low-dimensional model of the space of events. Namely, we consider the space that is shown at Figure 1. Here the lower straight line is the one dimensional analogy of our three dimensional Euclidean space, and the surface of cylinder (with the above mentioned straight line) is the analogy of the suggested model of the space-time.

Let us consider motions of free bodies in empty space that is motions without external fields of force. As in general relativity, we suggest that motions of such bodies take place along geodesic lines of the empty space-time, that is along shortest pathes between points of this space. Shortest pathes on the cylinder's surface are segments of the screw lines on this surface. Recall known relations for a screw line [9] (Figure 2).

$$
x=R \cos \tau, y=R \sin \tau, z=\frac{h}{2 \pi} \tau, l^{2}=(2 \pi R)^{2}+h^{2},
$$

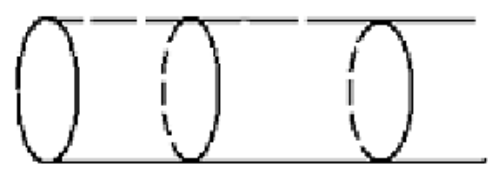

Figure 1. Examples of product of spaces.

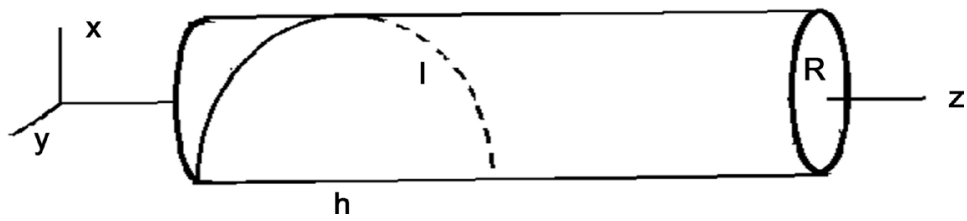

Figure 2. Geodesic screw line. 
where $R$ is the cylinder's radius, $h$ is a length of the pitch of screw, $l$ is a length of the one turn.

Consider the motion of a free body along the screw line with a small pitch of screw, when

$$
h \ll R .
$$

Then the displacement $X$ of this body in the Euclidean one dimensional space will be after $n$ turns

$$
X=n h .
$$

And the corresponding path $L$ along the screw line will be

$$
L=n 2 \pi R \text {. }
$$

It follows from (3) and (4) that

$$
X=\frac{h}{2 \pi R} L .
$$

Replace here $L$ by the variable $t$ with the dimensionality of time using relation

$$
L=c t
$$

where $c$ is the light velocity in vacuum. Then (5) can be rewritten as

$$
X=V t \text {, }
$$

where

$$
V=\frac{h}{2 \pi R} c
$$

We show now that above variable $t$ can be interpreted as a time-coordinate in the space of events. The coordinate $X$ in $(5,7)$ denotes the displacement of contact with one dimensional Euclidean space of the free body moving along the screw line in additional dimension (Figure 3).

This displacement looks as a sequence of periodical appearances and disappearances of this body in Euclidean space. But for sufficiently small $h$ these discontinuous displacements will look for observers in Euclidean space as continuous movement because of high frequency of this process. Indeed, according to (8), the frequency $\omega$ of this process for displacements with velocity $V$, corresponding to the pitch of screw $h$, equals

$$
\omega=\frac{V}{h}=\frac{c}{2 \pi R} \mathrm{~s}^{-1} .
$$

Let us suggest that $R$ has a microscopic dimension $R \sim 10^{-10} \mathrm{~m}$. Then

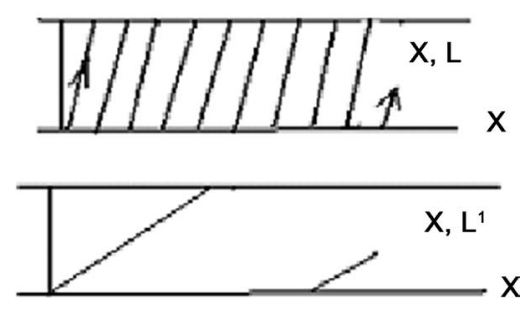

Figure 3. Discontinuous displacements in Euclidean space. 


$$
\omega \sim \frac{10^{8}}{10^{-10}} \mathrm{~s}^{-1}=10^{18} \mathrm{~s}^{-1} .
$$

Discontinuous displacements with such high frequencies will be detected as continuous ones by any of modern devices.

And here is the main point. If displacements of free bodies, described by (7), can be considered as continuous ones then (7) should be considered as the law of motion of free bodies in the Euclidean space, that is as the Galilei's inertia law. And this will be the case only if we identify variable $t$ in (7) as "time". The variable $t$ is defined by (6) through the length of free bodiy's trajectories along geodesic lines. In result, the geometrical interpretation of notion "time" looks as follows: time of a free body's displacement at distance $X$ in Euclidean space equals to the length $L$ of the trajectory of the same body in additional dimension divided by the light velocity $c$. Notice that according to $(2,8)$ this interpretation is valid for motions with nonrelativistic velocities $V \ll c$.

\section{Conclusions}

It is shown that within the framework of the suggested model of the space-time there is no fundamental difference between notions "space" and "time": both of them are represented by coordinates of the one space with specific geometry. It was shown also that there is a one-to-one correspondence between time coordinate (length of the body's displacement along the screw geodesic line) and the same body's displacement in the Euclidean space. This means that any kind of motion in Euclidean space can be chosen as a standard for measuring of time. Periodical movements (clocks) happened to be the most convenient in operation.

Notice in conclusion that suggested low-dimensional model of the space-time has mainly a methodical value. This work indicates at possible geometrical structure of the real space of events, namely, a topological product of our three-dimensional Euclidean space and the space with a topology of some closed manifold.

\section{References}

[1] Pauli, W. (1958) Theory of Relativity. Pergamon Press, Oxford.

[2] Dubrovin, B.A., Fomenko, A.T. and Novikov, S.P. (1985) Modern Geometry-Methods and Applications. Springer, Berlin.

https://doi.org/10.1007/978-1-4612-1100-6

[3] Olkhov, O.A. (2007) Geometrization of Quantum Mechanics. Journal of Physics. Conference Series, 67, Article ID: 012037. https://doi.org/10.1088/1742-6596/67/1/012037

[4] Olkhov, O.A. (2008) Geometrization of Classical Wave Fields. AIP Conferences Proceedings, 962, 316-321. https://doi.org/10.1063/1.2827325

[5] Olkhov, O.A. (2008) On the Possibility of Topological Interpretation of Quantum Mechanics. http://arXiv.org/abs/0802.2269

[6] Olkhov, O.A. (2014) Geometrical Approach in the Atomic Spectra Theory. Russian Journal of Physical Chemistry B, 8, 30-42. 
https://doi.org/10.1134/S199079311401014X

[7] Kaluza, Th. (1921) On the Unification Problem in Physics. Sitzungsberichte der Preussischen Akademie der Wissenschaften, 54, 966-972.

[8] Einstein, A. and Bergmann, P. (1938) On a Generalization of Kaluza's Theory of Electricity. Annals of Mathematics, 39, 685. https://doi.org/10.2307/1968642

[9] Rashewski, P.K. (1958) Differential Geometry. Nuka, Moscow. (In Russian)

Submit or recommend next manuscript to SCIRP and we will provide best service for you:

Accepting pre-submission inquiries through Email, Facebook, LinkedIn, Twitter, etc. A wide selection of journals (inclusive of 9 subjects, more than 200 journals)

Providing 24-hour high-quality service

User-friendly online submission system

Fair and swift peer-review system

Efficient typesetting and proofreading procedure

Display of the result of downloads and visits, as well as the number of cited articles Maximum dissemination of your research work

Submit your manuscript at: http://papersubmission.scirp.org/

Or contact jhepgc@scirp.org 